\title{
Real-world Data on the Efficacy and Safety of Ixazomib-based Therapy in Multiple Myeloma: A Single-center Study in China
}

This article was published in the following Dove Press journal: Cancer Management and Research

\author{
Kai Ding* \\ Hong $\mathrm{Yu}^{*}$ \\ Yuan-Yuan Shao \\ Li-Yan Li \\ Chao-Meng Wang \\ Jia Song \\ Li-Juan $\mathrm{Li}$ \\ Rong Fu
}

Department of Hematology, Tianjin Medical University General Hospital, Tianjin 300052, People's Republic of China

*These authors contributed equally to this work
Objective: To assess the short-term efficacy and safety of ixazomib in Chinese multiple myeloma (MM) patients in the real world.

Methods: Fifty-nine MM patients who received at least one cycle of ixazomib-based therapy between 1 June 2018 and 30 September 2019 were retrospectively analyzed in Tianjin Medical University General Hospital. Thirteen newly diagnosed MM (NDMM), 13 refractory/relapsed MM (RRMM) and 33 continuous therapy (27 bortezomib peripheral neuritis (PN) intolerant and six maintenance therapy) MM patients were included. The indicated overall response rate (ORR), time to overall response (TOR), and adverse events (AEs) were investigated.

Results: The ORR in NDMM was $76.9 \%$, with one complete response (CR), five very good partial response (VGPR), four partial response (PR), median PFS, and TOR were 122 (66272 ) days and 49 (22-108) days. The ORR in RRMM was $46.2 \%$, with one CR, two VGPR, three PR, median PFS, and TOR were 79 (28-169) days and 59 (23-88) days. The ORR in continuous therapy MM patients was $100 \%$, with nine stringent CR, $15 \mathrm{CR}$, six VGPR and three PR, median TOR was 75 (25-141) days. There were no significant differences regarding ORR between patients with cytogenetic high risk and standard risk in three subgroups (all $P>0.05$ ). The most frequent hematological AEs were anemia (13.6\%) and thrombocytopenia (10.2\%). The most common nonhematological AEs were PN (25.0\%) and diarrhea (13.6\%).

Conclusion: The real-world data demonstrated that ixazomib-based therapy was generally effective and safe in the short term for MM patients.

Keywords: multiple myeloma, ixazomib, real world, overall response rate, adverse events

\section{Introduction}

Multiple myeloma (MM) is a malignant clonal disease of plasma cells with poor prognosis and with a median survival of approximate five years, characterized by renal injury, osteolysis, hypercalcemia and anemia. ${ }^{1,2}$ Myeloma often affects elderly patients aged more than 65 years. ${ }^{3}$ A national population-based analysis data from 2012 to 2016 in China estimated that the standardized prevalence and incidence were 5.68 per 100,000 populations and 1.15 per 100,000 person-years, respectively. $^{4}$

Presently, improvement in the prognosis of MM was observed with the increasing application of novel agents such as proteasome inhibitor (PI) and immunomodulatory drugs. ${ }^{5}$ Both PI and immunomodulatory drugs have composed the backbone of newly 
diagnosed MM (NDMM) and relapse/refractory MM (RRMM) chemotherapy regimens. ${ }^{1,6}$ Continuous therapy and maintenance as the long-term treatment approaches in MM offers prolonged disease control and improved progression-free survival (PFS) and overall survival (OS) compared with traditional fixed-duration approaches. ${ }^{7}$ In order to optimize individual patient outcomes, the continuous therapy and maintenance treatment approaches are crucial for MM patients, which focus not only on efficacy and drug safety, but also on tolerability.

Ixazomib is the first oral bioavailable PI licensed by the China Food and Drug Administration for the treatment of MM in April 2018. Ixazomib is approved to be administered in combination with lenalidomide and dexamethasone (ixazomib-Rd) for the treatment of MM patients who have received at least one previous therapy. In the global Phase III TOURMALINE-MM1 study and China Continuation Study (CCS), ${ }^{8,9}$ ixazomib-Rd demonstrated a significant improvement in PFS vs placebo-Rd, with good safety and tolerability in RRMM patients. Similar results were shown in global phase I/II clinical trials of NDMM patients and a phase III clinical trial of maintenance treatment of MM patients. ${ }^{10,11}$ However, the realworld data on ixazomib-therapy for MM patients were seldom used in China.

The study aims to retrospectively evaluate the shortterm efficacy and safety of ixazomib in therapy of MM patients in the real-world clinical practice in China.

\section{Materials and Methods}

This single-center, retrospective, observational study was conducted in Tianjin Medical University General Hospital, and approved by the Ethics Committee of the hospital with the ethical number of IRB2020-WZ-051.

\section{Patients}

Fifty-nine patients hospitalized in the Hematology Department of Tianjin Medical University General Hospital between 1 June 2018 and 30 September 2019, with a diagnosis of NDMM or RRMM, or with bortezomib peripheral neuritis (PN) intolerant and maintenance therapy who received ixazomib-based chemotherapy were included. All patients were followed-up to October 30, 2019. Demographic characteristics and clinical data were collected and assessed. Patients were stratified by DurieSalmon stage, International Staging System (ISS) disease stage and revised-ISS (R-ISS) stage respectively, and the risk stratification based on International Myeloma
Working Group (IMWG) criteria - high risk mutation including del $(17 \mathrm{p}), \mathrm{t}(4,14)$ and $\mathrm{t}(14,16) .{ }^{11,12} \mathrm{RRMM}$ patients were identified by IMWG consensus.

\section{Treatment Regimens}

All patients received ixazomib on days 1, 8, and 15, in 28day cycles. Ixazomib dose was $4 \mathrm{mg}$ in patients with creatinine clearance (Clcr) $\geq 30 \mathrm{~mL} / \mathrm{min}$, and $3 \mathrm{mg}$ in patients with $\mathrm{Clcr}<30 \mathrm{~mL} / \mathrm{min}$ ). The number of patients received ixazomib at first, second, third, and fourth-line therapy was $19,30,8$, and 2 respectively. Ixazomib doublet- or triplet-drug regimens included: Id (ixazomib + dexamethasone $20 \mathrm{mg}$, days $1,2,8,9,15,16,22,23$, orally or intravenously) regimen; IRd (ixazomib + lenalidomide $25 \mathrm{mg}$, days 1-21, doses were modulated based on patients' Clcr + dexamethasone $20 \mathrm{mg}$, days 1, 2, 8, 9, 15, $16,22,23$, orally or intravenously); ITd (ixazomib + thalidomide $100 \mathrm{mg}$, days $1-28+$ dexamethasone $20 \mathrm{mg}$, days $1,2,8,9,15,16,22,23$, orally or intravenously); ICd (ixazomib + cyclophosphamide $300 \mathrm{mg} / \mathrm{m}^{2}$, intravenously, days $1,8,15,22+$ dexamethasone $20 \mathrm{mg}$, days $1,2,8,9$, $15,16,22,23$, orally or intravenously) and IAd (ixazomib + liposomal doxorubicin $40 \mathrm{mg}$, day $1+$ dexamethasone $20 \mathrm{mg}$, days $1,2,8,9,15,16,22,23$, orally or intravenously).

\section{Efficacy and Safety Evaluation}

The response/efficacy was evaluated using IMWG 2011 response criteria. ${ }^{13}$ Blood samples and $24 \mathrm{~h}$ urine were collected for MM evaluation at baseline and after each cycle of chemotherapy. Bone marrow examination was undertaken in all patients at baseline, the clonal evolution was investigated at the time of disease progression, and minimal residual disease (MRD) was evaluated at the time of response to $\geq$ complete remission (CR). The outcomes included stringent complete remission (sCR), CR, very good partial remission (VGPR), PR, minimal remission (MR), stable disease (SD), progressive disease (PD) and overall response rate (ORR: $\geq \mathrm{PR}$ ). Adverse events (AEs) were assessed using the National Cancer Institute Common Toxicity Criteria for AEs, version 5.0.

\section{Statistical Analysis}

Baseline characteristics were assessed with descriptive statistical analysis; the mean and standard deviation (SD) or median (range) are presented for continuous variables and frequency distribution (n, \%) presented for categorical variables and compared using the chi-squared test. All tests were 
two-sided and a level of $P<0.05$ served as the threshold for statistical significance. All statistical analyses were performed in SPSS statistical software package (SPSS version 19.0, IBM Corporation, Armonk, NY, USA).

\section{Results}

\section{Baseline Characteristics and Treatment}

Among 59 MM patients, 13 were (22.0\%) NDMM, 13 (22.0\%) RRMM (four relapse, three refractory, six relapse and refractory), and 33 (55.9\%) continuous therapy (all better than PR, six patients continued to maintenance after $\mathrm{CR}$ and 27 bortezomib PN intolerant). The median age was $67(47-84)$ years and $59.3 \%$ of patients were male (Table 1).

\section{Efficacy NDMM Patients}

The median follow-up from first ixazomib dose was three (1-11) months. The ORR was $76.9 \%$ (10/13), with one patient obtaining CR, five VGPR, and four PR. MRD was positive in the patient with $\mathrm{CR}$. The median time to response was 49 (22-108) days (Table 2). The median PFS was 122 (66-272) days. In 13 patients (four younger and nine older than 65 years) the ORR were $100 \%$ and $66.7 \%$ respectively, the median number of cycles to response of ixazomib were 2.5 (1-4) and 2.0 (1-4) respectively. In eight frail patients, all received Id regimens, the ORR was $62.5 \%$, with three VGPR, two PR (Supporting information in Table S1).

\section{RRMM Patients}

The median follow-up was four (2-10) months. The ORR in RRMM patients was $46.2 \%$ (6/13), with one CR, two VGPR, three PR. MRD was positive in the patient with CR. The median time to response was 59 (23-88) days (Table 2). The median PFS was 79 (28-169) days. Of them, six patients received IRd regimen, three IAd, three ICd and one ITd regimen. The ORR in nine bortezomib resistant patients was $33.3 \%$ (3/9), with one VGPR (received IAD), two PR (received IAD and IRD). The median response time was 70 (28-112) days.

\section{Continuous Therapy Patients}

The median follow-up was six (2-11) months. The ORR in 33 continuous therapy patients was $100 \%$, with nine sCR, $15 \mathrm{CR}$, six VGPR, three PR. MRD was negative in three of nine patients with sCR and six of 15 patients with $\mathrm{CR}$. The median time to response was 75 (25-141) days (Table 2).
Table I Baseline Characteristics of 59 MM Patients

\begin{tabular}{|c|c|}
\hline Baseline Characteristics & n (\%) \\
\hline \multicolumn{2}{|l|}{ Age } \\
\hline Median (range), years & $67(47-84)$ \\
\hline$<65$ years & $26(44.1)$ \\
\hline$\geq 65-75$ years & $27(45.8)$ \\
\hline$>75$ years & $6(10.2)$ \\
\hline \multicolumn{2}{|l|}{ Gender } \\
\hline Male & $35(59.3)$ \\
\hline Female & $24(40.7)$ \\
\hline NDMM & $13(22.0)$ \\
\hline Continued to therapy & $33(55.9)$ \\
\hline Bortezomib PN-intolerant ( $\geq \mathrm{PR})$ & $27(45.8)$ \\
\hline RRMM & $13(22.0)$ \\
\hline Relapse & $4(6.8)$ \\
\hline Refractory & $3(5.1)$ \\
\hline Relapse and refractory & $6(10.2)$ \\
\hline \multicolumn{2}{|l|}{ ECOG PS } \\
\hline $0-1$ & $12(20.3)$ \\
\hline 2 & $19(32.2)$ \\
\hline$\geq 3$ & $28(47.5)$ \\
\hline \multicolumn{2}{|l|}{ ISS disease stage } \\
\hline 1 & $8(15.7)$ \\
\hline$\|$ & $13(22.0)$ \\
\hline III & $38(64.4)$ \\
\hline \multicolumn{2}{|l|}{ R-ISS disease stage } \\
\hline 1 & $6(11.8)$ \\
\hline ॥ & $30(50.8)$ \\
\hline III & $23(39.0)$ \\
\hline \multicolumn{2}{|l|}{ Durie-Salmon stage } \\
\hline 1 & $3(5.1)$ \\
\hline ॥ & $10(16.9)$ \\
\hline III & $46(78.0)$ \\
\hline M-protein subtype $\lg G \lambda$ & $19(32.2)$ \\
\hline $\lg G$ & $32(54.2)$ \\
\hline $\lg A$ & $12(20.3)$ \\
\hline $\lg E$ & $\mathrm{I}(\mathrm{I} .7)$ \\
\hline $\lg G / A$ & I (I.7) \\
\hline$\lambda$ light chain & II (I8.6) \\
\hline$\kappa$ light chain & $2(3.4)$ \\
\hline \multicolumn{2}{|l|}{$\mathrm{ClCr}, \mathrm{mL} / \mathrm{min}$} \\
\hline$<30$ & $12(20.30)$ \\
\hline $30-<60$ & $13(22.0)$ \\
\hline $60-<90$ & $13(22.0)$ \\
\hline$\geq 90$ & $21(35.6)$ \\
\hline \multicolumn{2}{|l|}{ IMWG myeloma frailty status } \\
\hline Fit & $8(13.6)$ \\
\hline Intermediate & $30(50.8)$ \\
\hline Frail & $21(35.6)$ \\
\hline
\end{tabular}

(Continued) 
Table I (Continued).

\begin{tabular}{|l|l|}
\hline Baseline Characteristics & n (\%) \\
\hline $\begin{array}{l}\text { Cytogenetic profile } \\
\text { Standard risk } \\
\text { High risk }\end{array}$ & $48(8 I .4)$ \\
\hline $\begin{array}{l}\text { Prior treatment (except NDMM) } \\
\text { Median cycle of treatment (range) }\end{array}$ & $1 \mathrm{I} 8.6)$ \\
PI & $3(1-14)$ \\
IMiD & $17(37.0)$ \\
PI+IMiD & $3(6.5)$ \\
\hline
\end{tabular}

Note: ${ }^{a}$ High-risk cytogenetic was defined as any of dell7p, $t(4 ; 14)$, or $t(14 ; 16)$. Abbreviations: NDMM, newly diagnosed multiple myeloma; RRMM, refractory/ relapsed multiple myeloma; ECOG PS, Eastern Cooperative Oncology Group performance status; ISS, International Staging System; R-ISS, revised International Staging System; $\mathrm{ClCr}$, creatinine clearance rate; PI, proteasome inhibitor; IMiD, immunomodulatory drug.

Twenty-seven patients responded better than PR after prior triplet- bortezomib-based therapy, however, they were intolerant to bortezomib PN; Of them, 10 patients $(37.0 \%)$ received improved response, the median number of treatment cycles was three (one to five). Afterwards, these patients with bortezomib PN intolerant substituted ixazomib therapy for bortezomib, of whom 16 patients received IRd, five IAd, four ICd and two ITd. In other six patients received Id regimen as maintenance therapy, the median

Table 2 Efficacy Evaluation of 59 MM Patients

\begin{tabular}{|l|l|l|l|}
\hline Items & $\begin{array}{l}\text { NDMM } \\
\text { (n=13) } \\
\text { n (\%) }\end{array}$ & $\begin{array}{l}\text { RRMM } \\
\text { (n=13) } \\
\text { n (\%) }\end{array}$ & $\begin{array}{l}\text { Continuing } \\
\text { Therapy } \\
\text { (n=33) n (\%) }\end{array}$ \\
\hline $\begin{array}{l}\text { Median follow-up (months) } \\
\text { Median time to response }\end{array}$ & $3(1-1 \mathrm{I})$ & $4(2-10)$ & $6(2-1 \mathrm{I})$ \\
(days) & $108)$ & $59(23-$ & $75(25-14 I)$ \\
SCR & - & - & $9(27.3)$ \\
CR & $1(7.7)$ & $1(7.7)$ & $15(45.5)$ \\
VGPR & $5(38.5)$ & $2(15.4)$ & $6(18.2)$ \\
PR & $4(30.8)$ & $3(23.1)$ & $3(9.1)$ \\
MR & $1(7.7)$ & $0(0.0)$ & $0(0,0)$ \\
SD & $1(7.7)$ & $1(7.7)$ & $0(0,0)$ \\
PD & $1(7.7)$ & $6(46.2)$ & $0(0,0)$ \\
\hline ORR & $10(76.9)$ & $6(46.2)$ & $33(100)$ \\
$<65$ years & $4(100.0)$ & $9(69.2)$ & $25(75.8)$ \\
$\quad \geq 65$ years & $6(66.7)$ & $4(30.8)$ & $8(24.2)$ \\
Cytogenetic high risk & $3(100.0)$ & $2(50.0)$ & $4(100.0)$ \\
Cytogenetic standard risk & $7(70.0)$ & $4(44.4)$ & $29(100.0)$ \\
\hline
\end{tabular}

Abbreviations: NDMM, newly diagnosed multiple myeloma; RRMM, refractory/ relapsed multiple myeloma; $\mathrm{sCR}$, stringent complete remission; $\mathrm{CR}$, complete remission; VGPR, very good partial remission; PR, partial remission; $M R$, minimal remission; SD, stable disease; $P D$, progressive disease; ORR, overall response rate ( $\geq P R$ ). treatment duration was four (two to six) cycles, two (33.3\%) received improved response to $\mathrm{sCR}$, and all responded better than $\mathrm{CR}$ after $\geq 4$ cycles of prior therapies (two patients received PAd, one VRd and three RAd). At the end of follow-up, no relapse or PDs were reported. Across the entire study, the median PFS was not reached for patients with continuous therapy.

\section{Subgroup Analysis in Different Cytogenetic Risk Patients}

The ORR between patients with cytogenetically high risk and standard risk were respectively NDMM (100\%, 70\%), RRMM $(50.0 \%, 44.4 \%)$, continuous therapy $(100 \%$, $100 \%$ ). The differences between the two groups were all without statistical significance (all $P>0.05$ ). The results were shown in Table 2.

$13.6 \%$ of patients suffered gene lq21 abnormality, $8.5 \%$ undergone gene $17 \mathrm{p}$ - mutation. The proportions of gene $\mathrm{t}(14,16), \mathrm{t}(11,14)$ and $\mathrm{t}(4,14)$ abnormalities were $5.1 \%, 5.1 \%$ and $3.4 \%$, respectively (Table 3 ).

\section{Safety}

The most frequently reported hematological AEs were anemia (13.6\%) and thrombocytopenia (10.2\%). The most common nonhematological AEs were peripheral neuritis $(25.0 \%)$ and diarrhea (13.6\%). Grade 3/4 AEs were reported in nine cases $(15.3 \%)$. The most commonly reported grade 3/4 AEs were thrombocytopenia (3.4\%) and cardiac events $(3.4 \%)$. The results were shown in Table 4. However, these AEs can be relieved and improved via symptomatic treatment.

\section{Discussion}

Ixazomib, when combined with dexamethasone and lenalidomide, has shorter dissociation half-life, better pharmacokinetics, more powerful anti-MM efficacy, even in bortezomib-resistant cells, compared with first generation

Table 3 The Cytogenetically Risk Profile of All 59 Patients

\begin{tabular}{|l|l|}
\hline Cytogenetic Abnormality & n (\%) \\
\hline Iq2I & $8(13.6)$ \\
I7p- & $5(8.5)$ \\
$\mathrm{t}(I 4,16)$ & $3(5 . I)$ \\
$\mathrm{t}(I I, \mid 4)$ & $3(5.1)$ \\
$\mathrm{t}(4,14)$ & $2(3.4)$ \\
Other mutations & $7(11.9)$ \\
More than two mutations & $12(20.3)$ \\
Negative & $19(32.2)$ \\
\hline
\end{tabular}


Table 4 Safety Analysis of 59 MM Patients

\begin{tabular}{|l|l|l|}
\hline & Any Grade & Grade 3/4 \\
\hline Hematological adverse events & & \\
Anemia & $8(13.6)$ & $\mathrm{I}(\mathrm{I} .7)$ \\
Thrombocytopenia & $6(10.2)$ & $2(3.4)$ \\
Neutropenia & $5(8.5)$ & - \\
\hline Nonhematological adverse events & & \\
PN $N^{\mathrm{a}}$ (32 patients) & $8(25.0)$ & $\mathrm{I}(3 . \mathrm{I})$ \\
Diarrhea & $8(13.6)$ & $\mathrm{I}(\mathrm{I} .7)$ \\
Infection & $5(8.5)$ & - \\
Cardiac events & $4(6.8)$ & $2(3.4)$ \\
Liver dysfunction & $2(3.4)$ & - \\
Nausea and vomit & $2(3.4)$ & - \\
Skin rash & $2(3.4)$ & $\mathrm{I}(\mathrm{I} .7)$ \\
Fatigue & $2(3.4)$ & - \\
Edema & $\mathrm{I}(\mathrm{I} .7)$ & - \\
Cerebropathy & $\mathrm{I}(\mathrm{I} .7)$ & $\mathrm{I}(\mathrm{I}) \mathrm{7})$ \\
Constipation & $\mathrm{I}(\mathrm{I} .7)$ & - \\
\hline
\end{tabular}

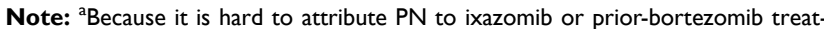
ment, the 27 bortezomib PN intolerant patients in continuing therapy group were excluded here, and analyzed separately in the discussion part.

Abbreviation: PN, peripheral neuritis.

PI. ${ }^{14,15}$ A few studies indicated that ixazomib monotherapy, or doublet-/triplet-drug regimens with dexamethasone or/ and lenalidomide, were effective in patients with NDMM, ${ }^{10}$ RRMM, ${ }^{8,9}$ and MM maintenance therapy after autologous stem cell transplantation. ${ }^{11}$ In this study, $56.0 \%$ of MM patients were older than 65 years, $10.2 \%$ were older than 75 years. As known, age is one of the most important prognostic factors in the treatment of $\mathrm{MM} .{ }^{16} \mathrm{~A}$ published study reported that approximately one-third of MM patients were older than 75 years at the time of diagnosis. ${ }^{17}$

In our study, the ORR was $76.9 \%$ in NDMM. Moreover, the ORR in patients who were younger than 65 years was higher than those who were older (100\% vs $66.7 \%)$. It was possible that the frail patients in the group of younger than 65 years were fewer than those in the group of older than 65 years (50.0\% vs $66.7 \%)$. A published phase II study demonstrated that the ORR was $92 \%$ in patients with previously untreated MM with IRd regimen. ${ }^{18}$ However, 92\% NDMM patients were treated with Id doublet regimens in our study. In addition, the median PFS was almost four months, which was much shorter than the 35.4 months reported in a long-term follow-up study in patients with NDMM. ${ }^{19}$ Different study population, treatment regimen, treatment duration and variety study design probably led to differences in multiple studies.

This study showed that the ORR was $46.2 \%$ of RRMM patients and the median time to response was 59 days. The results were similar with a published abstract of the realworld study that included 27 Chinese patients with ixazomib-based therapy in MM (with 12 NDMM and 15 RRMM) which demonstrated that the ORR of RRMM was $53.3 \%$ and the median time to response was 1.76 months. ${ }^{20}$ Moreover, a published retrospectively realworld study which focused on the effectiveness and safety of lenalidomide plus dexamethasone (RD) in 546 patients with RRMM at 25 university hospitals in South Korea between October 2009 and December 2016 found that the ORR of RRMM patients who received median seven cycles of RD was $64.2 \%$, with CR $13.1 \%$ and VGPR $19.9 \%$. $^{21}$ The rate in our study was slightly lower than that demonstrated in the Korean study. In addition, the ORR in our study was significantly lower than $78 \%$, which was reported for RRMM patients in the TOURMALINE-MM1 study. ${ }^{8}$ In our study, $76.9 \%$ patients received ixazomib as more than third-line therapy, and $69.2 \%$ of patients were bortezomibresistant who were excluded in the TOURMALINE-MM1 trial, which might lead to a lower ORR. Furthermore, the median PFS was 2.6 months in our study, however, median PFS have been reported as 10-18 months in other retrospective studies and 6.7 months in a phase 3 randomized controlled trial. ${ }^{9,21}$ The results varied from kinds of therapeutic drugs and treatment duration, different diagnosis and treatment environment, diverse health status of patients, and variety of sample size. The possible causes need further analysis.

Thirty-seven percent of continuing therapy patients received improved response after a median three cycles of ixazomib therapy in our study. The ORR of patients with continuous therapy was $100 \%$, the median response time was 75 days. However, most $(81.8 \%)$ of them were bortezomib PN-intolerant. Afterwards, the physician substituted bortezomib for ixazomib because of its slight PNadverse events. ${ }^{8,9}$ An open-label phase 1/2 study suggested that ixazomib-based therapy had improvement in depth of response in patients continuing with maintenance therapy. $^{18}$

The most common hematological AEs were thrombocytopenia (13.6\%) and anemia (15.3\%) in our study which were in line with the incidences (18.4\% and $8.2 \%)$ reported in a real-world study of lenalidomide and dexamethasone therapy for RRMM patients. ${ }^{21}$ Despite transient blood transfusion needed in three patients, no discontinuation was reported because of hematological AEs. PN is the most common AE of first-generation PI, the incidence was approximate $40 \%,{ }^{22}$ which decreased the life quality of 
patients and treatment compliance. Nevertheless, our study reported lower incidence $(25.0 \%)$ and less severity (grade $3 / 43.1 \%$ ) of PN. This may have been due to missing data and small sample size. In 27 PN-intolerant but sensitive patients, $11.1 \%$ had PN aggravated, 29.6\% had PN stable and $59.3 \%$ had PN relieved gradually, which suggested good safety of PN with ixazomib therapy.

In our study, 86.4\% (51/59) of patients (11 of 13 patients with NDMM could not be included, four patients with myocardial amyloidosis and $\mathrm{ECOG} \geq 3$, five with $\mathrm{ECOG} \geq 3$, and two with $\mathrm{Clcr} \leq 30 \mathrm{~mL} / \mathrm{min} ; 13$ patients with RRMM could not be enrolled, 10 patients with greater than third-line treatment and three patients with bortezomib resistance; among 33 patients with continuous treatment, 27 patients with bortezomib PN intolerance had grade $1 / 2$ pain could not be enrolled) were ineligible for the inclusion criteria in the TOURMALINE-MM1 study, who composed a large part of MM patients in China, similarly worldwide. Our study also has some limitations. Firstly, it was retrospective and had a small sample size in a single institution. Secondly, the follow-up was too short to measure OS and entire PFS. Thirdly, comparisons between different treatments were not conducted because of limited numbers of patients. In addition, some information might not be recorded and lost due to the retrospective design. However, the advantage of this study was that NDMM, RRMM and continuous therapy patients were all enrolled and stratified analysis was performed. We will continue to have a longer follow-up and continue to expand the sample size in each subgroup. Therefore, our study demonstrates the efficacy and safety of ixazomib-based therapy in the real-world clinical setting and should provide useful information to physicians treating patients with MM in clinical practice.

\section{Conclusions}

The study indicated that ixazomib-based treatments were proved to be effective in the short term for MM patients, also well-tolerated and safe in Chinese real-world practices. And the longer follow-up, larger sample size and case-controlled clinical study of ixazomib-based therapy for MM patients in the real-world also deserved to be performed in the future.

\section{Ethical Approval}

The study was approved by the Ethics Committee of Tianjin Medical University General Hospital, with the ethical number of IRB2020-WZ-051. All authors declare that patient data were covered confidentiality. All authors affirm that the guidelines outlined in the Declaration of Helsinki were met.

\section{Consent to Participate}

The need for informed consent was waived by the Ethics Committee of Tianjin Medical University General Hospital because the retrospective study involved existing data and records at the time of investigation, and the study has been performed in accordance with the ethical standards as laid down in the 1964 Declaration of Helsinki.

\section{Acknowledgments}

We thank all participants and their families, as well as the physicians, nurses, study coordinators, and research staff for participation in the study. We also thank Ms Hui Liu and Prof./Ms Rong Fu for the study design, data analysis and editorial assistance; Ms Hui Liu and Mr Zhao-yun Liu for professional medical writing assistance.

\section{Disclosure}

The authors report no conflicts of interest in this work.

\section{References}

1. Garderet L, Iacobelli S, Moreau P, et al. Superiority of the triple combination of bortezomib-thalidomide-dexamethasone over the dual combination of thalidomide-dexamethasone in patients with multiple myeloma progressing or relapsing after autologous transplantation: the MMVAR/IFM 2005-04 Randomized Phase III Trial from the Chronic Leukemia Working Party of the European Group for Blood and Marrow Transplantation. J Clin Oncol. 2012;30(20):2475-2482. doi:10.1200/JCO.2011.37.4918

2. Kumar SK, Rajkumar V, Kyle RA, et al. Multiple myeloma. Nat Rev Dis Primers. 2017;3(1):17046. doi:10.1038/nrdp.2017.46

3. Knauf W, Dingeldein G, Schlag R, et al. First-line therapy with Bendamustine/Prednisone/Bortezomib (BPV) - A GMMG trial for non-transplant eligible symptomatic multiple myeloma patients. Eur J Haematol. 2020;105(2):116-125. doi:10.1111/ejh.13409

4. Wang S, Xu L, Feng J, et al. Prevalence and Incidence of Multiple Myeloma in Urban Area in China: A National Population-Based Analysis. Front Oncol. 2019;9:1513. doi:10.3389/fonc.2019.01513

5. Kumar SK, Dispenzieri A, Lacy MQ, et al. Continued improvement in survival in multiple myeloma: changes in early mortality and outcomes in older patients. Leukemia. 2014;28(5):1122-1128. doi:10. 1038/leu.2013.313

6. Dimopoulos M, Spencer A, Attal M, et al. Lenalidomide plus dexamethasone for relapsed or refractory multiple myeloma. $N$ Engl J Med. 2007;357(21):2123-2132. doi:10.1056/NEJMoa070594

7. Dimopoulos MA, Jakubowiak AJ, McCarthy PL, et al. Developments in continuous therapy and maintenance treatment approaches for patients with newly diagnosed multiple myeloma. Blood Cancer J. 2020;10(2):2. doi:10.1038/s41408-020-0273-x

8. Moreau P, Masszi T, Grzasko N, et al. Oral Ixazomib, Lenalidomide, and Dexamethasone for Multiple Myeloma. $N$ Engl J Med. 2016;374 (17):1621-1634. doi:10.1056/NEJMoa1516282

9. Hou J, Jin J, Xu Y, et al. Randomized, double-blind, placebo-controlled phase III study of ixazomib plus lenalidomide-dexamethasone in patients with relapsed/refractory multiple myeloma: china Continuation study. J Hematol Oncol. 2017;10(1):137. doi:10.1186/ s13045-017-0501-4 
10. Kumar SK, Buadi FK, LaPlant B, et al. Phase 1/2 trial of ixazomib, cyclophosphamide and dexamethasone in patients with previously untreated symptomatic multiple myeloma. Blood Cancer J. 2018;8 (8):70. doi:10.1038/s41408-018-0106-3

11. Dimopoulos MA, Gay F, Schjesvold F, et al. Oral ixazomib maintenance following autologous stem cell transplantation (TOURMALINE-MM3): a double-blind, randomised, placebo-controlled phase 3 trial. Lancet. 2019;393(10168):253-264. doi:10.1016/ S0140-6736(18)33003-4

12. Anderson KC, Kyle RA, Rajkumar SV, et al. Clinically relevant end points and new drug approvals for myeloma. Leukemia. 2008;22 (2):231-239. doi:10.1038/sj.leu.2405016

13. Kumar S, Paiva B, Anderson $\mathrm{KC}$, et al. International Myeloma Working Group consensus criteria for response and minimal residual disease assessment in multiple myeloma. Lancet Oncol. 2016;17(8): e328-e346. doi:10.1016/S1470-2045(16)30206-6

14. Kupperman E, Lee EC, Cao Y, et al. Evaluation of the proteasome inhibitor MLN9708 in preclinical models of human cancer. Cancer Res. 2010;70(5):1970-1980. doi:10.1158/0008-5472.CAN-09-2766

15. Chauhan D, Tian Z, Zhou B, et al. In vitro and in vivo selective antitumor activity of a novel orally bioavailable proteasome inhibitor MLN9708 against multiple myeloma cells. Clin Cancer Res. 2011;17(16):53115321. doi:10.1158/1078-0432.CCR-11-0476

16. Qian X, Chen H, Xia J, Wang J, Zhou X, Guo H. Real-World Clinical Outcomes in Elderly Chinese Patients with Multiple Myeloma: A Single-Center Experience. Med Sci Monit. 2018;24:5887-5893. doi:10.12659/MSM.907588
17. Zweegman S, Engelhardt M, Larocca A. 'Aging ESo, Hematology. Elderly patients with multiple myeloma: towards a frailty approach? Curr Opin Oncol. 2017;29(5):315-321. doi:10.1097/CCO.0000000 000000395

18. Kumar SK, Berdeja JG, Niesvizky R, et al. Safety and tolerability of ixazomib, an oral proteasome inhibitor, in combination with lenalidomide and dexamethasone in patients with previously untreated multiple myeloma: an open-label phase 1/2 study. Lancet Oncol. 2014;15(13):1503-1512. doi:10.1016/S1470-2045(14)71125-8

19. Kumar SK, Berdeja JG, Niesvizky R, et al. Ixazomib, lenalidomide, and dexamethasone in patients with newly diagnosed multiple myeloma: long-term follow-up including ixazomib maintenance. Leukemia. 2019;33(7):1736-1746. doi:10.1038/s41375-019-0384-1

20. Li J, Zhuang J, Wei Z, Ji L, Cheng Z, Liu P. Real World Data on the Efficacy and Safety of Ixazomib Based Therapy in Multiple Myeloma: an Observational Study from China. Clin Lymphoma Myeloma Leuk. 2019;19(10):e265. doi:10.1016/j.clml.2019.09.438

21. Jo JC, Lee HS, Kim K, et al. The effectiveness and safety of lenalidomide and dexamethasone in patients with relapsed/refractory multiple myeloma in real-world clinical practice: a study of the Korean Multiple Myeloma Working Party (KMMWP-151 study). Ann Hematol. 2020;99(2):309-319. doi:10.1007/s00277-019-03904-7

22. Morawska M, Grzasko N, Kostyra M, Wojciechowicz J, Hus M. Therapy-related peripheral neuropathy in multiple myeloma patients. Hematol Oncol. 2015;33(4):113-119. doi:10.1002/hon.2149

\section{Publish your work in this journal}

Cancer Management and Research is an international, peer-reviewed open access journal focusing on cancer research and the optimal use of preventative and integrated treatment interventions to achieve improved outcomes, enhanced survival and quality of life for the cancer patient.
The manuscript management system is completely online and includes a very quick and fair peer-review system, which is all easy to use. Visit http://www.dovepress.com/testimonials.php to read real quotes from published authors. 